\title{
Techniques for the Extraction of Vibrational Signature: A New Method of Pottery Shard Identification
}

\author{
Baxton R. Chen \\ Foundation for the Advancement of Anthropology \& History, Menlo Park, CA, USA \\ Email: Baxraychen@me.com
}

How to cite this paper: Chen, B. R. (2018). Techniques for the Extraction of Vibrational Signature: A New Method of Pottery Shard Identification. Archaeological Discovery, 6, 271-277.

https://doi.org/10.4236/ad.2018.63013

Received: April 23, 2018

Accepted: June 16, 2018

Published: June 19, 2018

Copyright $\odot 2018$ by author and Scientific Research Publishing Inc. This work is licensed under the Creative Commons Attribution International License (CC BY 4.0).

http://creativecommons.org/licenses/by/4.0/

\begin{abstract}
Pottery shards are conventionally classified based on their color, surface texture, density, thickness, curvature, material, and shape. We previously reported a method of identifying pottery shards based on their vibrational characteristics with ultrasound stimulation. We now detail the experimental procedure necessary for the extraction of such signatures. With the method of rapid and inexpensive vibrational signature extraction and comparison to a known database library, the technique provides a potential method of onsite shard identification.
\end{abstract}

\section{Keywords}

Pottery, Vibration, Identification, Algorithm, Signature

\section{Introduction}

The history of pottery echoes the history of human civilization. Ever since fire was first used, man has found the hardened soil around the fire useful for molding into figurines or containers (Renfrew \& Bahn, 1991). As mankind evolved and mastered fire, different cultures around the world independently developed their own sophisticated styles of pottery, often with distinctive shapes, colors, and styles based on local soil and other materials, technology, and artistic expression. The variety of pottery developed has proven helpful when studying past cultures and civilizations. Due to the nearly indestructible hardiness of pottery shards, they have been consistently uncovered from many excavation sites. Overtime, the excavation of items of a particular pottery style alongside other known objects helped to establish pottery shards as a useful tool to chronologically date excavation sites. Pottery shard identification has thus become an im- 
portant discipline in the field of Archaeology. Archaeologists have traditionally relied on color, surface texture, density, thickness, curvature, material, and shape to identify pottery shards (Historical Archaeology at the Florida Museum of Natural History, 2018; Hunt, 2017). This method of pottery shard ientification requires years of training, and disagreements among experts are not unusual. It would be useful to have a physical method of identifying pottery shards.

As a general principle, all materials vibrate when exposed to sound waves (MIT News, 2014). Pottery is made of dense material that transmits sound and vibrations easily, with distinctive harmonic waves that are characteristic of the material since soils from different parts of the world have different characteristics, they possess unique vibrational signatures (Parikh et al., 2014; Gazetas, 1982). For example, different concentrations of clay soil can influence vibrational signatures (Kitovas, Stelmokaitis, \& Doroševas, 2016). The unique vibrational characteristics of North American soil has long been documented (Jacobsen, 1930), and recent studies list European soil vibrational patterns (Eddine, Lenti, \& Semblat, 2017). The vibrational signatures of soil around Scotland were also carefully catalogued in a recent report detailing the responses to infrared and x-ray (Robertson, Shand, \& Perez-Fernandez, 2018). Since pottery made in each region comprises different soil compositions, each has unique vibrational characteristics. We previously reported using ultrasound-triggered vibrational analysis to automatically differentiate pottery (Chen, 2017). We now detail the technique necessary for extracting such vibrational signatures.

\section{Materials and Methods}

The system consists of a source for energy stimulation, a holding system for the pottery shard, a system for vibration detection, and a system for vibrational analysis. A number of energy sources have been utilized, including radiofrequency, ultrasound, or mechanical force. At the simplest level, one can strike a shard of pottery with a tuning fork or a small jeweler's hammer to generate a simple and reproducible stimulus. The stimulus is absorbed by the pottery shard, which will then vibrate in accordance to its internal harmonic frequency, which is characteristic of the pottery composition. Alternatively, one can use signal sources such as medical grade Dermatology treatment machines, such as Mini RF Bipolar Radio Frequency (Figure 1(a); UPC code 710280118370, Mychway, Hong Kong, China) or D’Arsonval High Frequency Device (Figure 1(b); Napalabscience, China). At a higher price point, one can use medical grade ultrasound machines such as the VScan pocket ultrasound (GE Healthcare, Little Chalfont, United Kingdom). This type of device produces extremely precise frequencies of ultrasound that are routinely used for medical imaging, and generates high resolution signatures.

The pottery shard specimen is held in place with a simple clamp, which may or may not need to be anchored to a stand for stability. A number of different clamps can be used to hold the pottery shard. The most important precaution is 


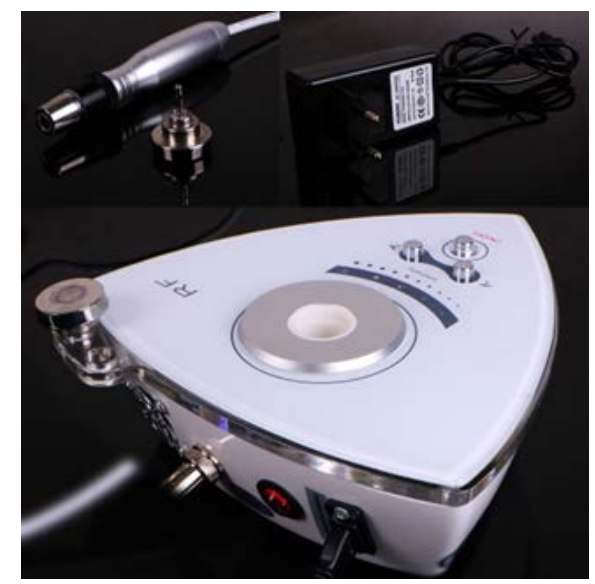

(a)

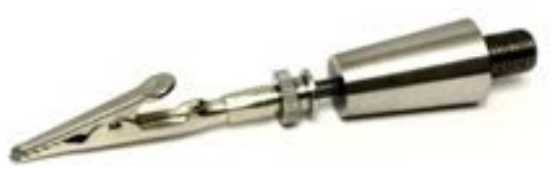

(c)

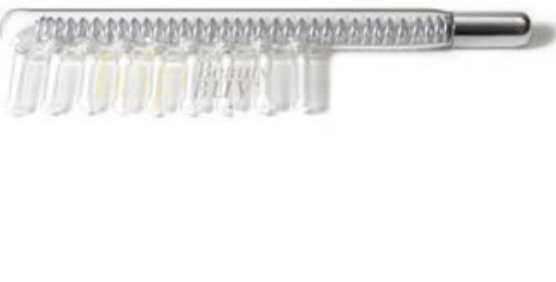

(b)

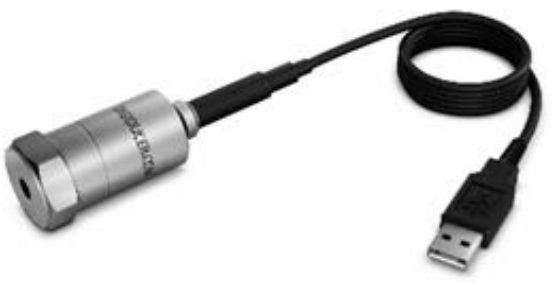

(d)

Figure 1. (a) Mini RF Bipolar Radio Frequency; (b) D’Arsonval High Frequency Device; (c) Screw-mounted micro-clamp; (d) USB Digital Accelerometer.

to find a clamp that can hold the shard tightly so as to prevent the shard's wobbling, which can produce distracting background vibrations. The clamp can reduce vibration at the point of contact, soit is important to choose a clamp with as small of a clamp footprint as possible in order to prevent damping of the vibrational signature. One should be sure to use clamps that are non-crushing in order to prevent damage to the pottery shards.

The vibration detection system consists of an USB Digital Accelerometer (Model 333D01, Digiducer, Mission Viejo, CA). The accelerometer is connected to the pottery shard via a screw-mounted micro-clamp (Figure 1(c); SCP-MICRO, SnakeClamp Products, Riner, VA). The small profile clamp can transmit signals from the pottery to the accelerometer with minimal signal loss or distortion of signal integrity. The Accelerometer is then connected to an iPad, iPad Mini, or to an iPhone via the Lightning-to-USB Camera Adapter (Apple, Cupertino, CA).

The vibrational analysis system utilizes apps such asVibroChecker Pro (ACE Control Inc, Farmington, MI) or SignalScope (Faber Acoustical, LLC, Lehi, Utah). Both softwares can interpret the signal received from the accelerometer and display them as waveforms that can be easily visualized and recorded.

\section{Results}

The signal source and the accelerometer are placed approximately one inch apart for standardized signal capture (Figure 2). Pending on the source of stimulation 


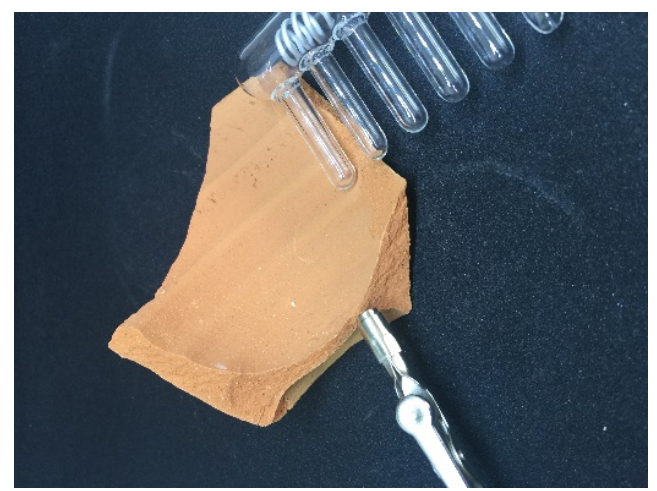

Figure 2. The signal source and the accelerometer are placed approximately one inch apart for standardized signal capture.

and the shape of the pottery shard, additional clamps may be necessary for sample stabilization. It is important to record the exact signal-capturing setup, as the setup may interfere with the vibrational signal. In order to facilitate accurate comparisons, all pottery shards in the database library should be tested and recorded with specified settings.

Here we show the results from a commercially available, common 20th century unglazed, lead free pottery shard made in Mexico (Figure 3(a)). The recording is shown in Figure 3(b). One can see the vibrational profile with spikes in the $9 \mathrm{~K}, 6 \mathrm{~K}$, and $1 \mathrm{~K} \mathrm{~Hz}$ range. In addition, one sees low frequency vibration in the below $10 \mathrm{~Hz}$ range. It is important to record and compare the full spectrum of spikes, including the low frequency vibrations plateau. The plateau represents the summation of low frequency spikes which may not be apparent as individual spikes, yet represent a part of the pottery shard's vibrational profile.

Figure 4(a) shows a similar 20th century glazed pottery piece, also from Mexico. The corresponding recording in Figure $4(\mathrm{~b})$ showed spikes in the $4 \mathrm{~K}, 1 \mathrm{~K}$, and $300 \mathrm{~Hz}$ range. In addition, one also sees low frequency vibration, although this one has a peak vibration frequency in the $30 \mathrm{~Hz}$ range. These two pottery samples are from similar regions in Mexico, but one can see the vibrational profiles can differentiate based upon different techniques of preparation.

\section{Discussion}

Big data analytics have had significant recent advances, with many algorithms developed for face recognition as a means of classifying photos uploaded on social media networks. The extraction of a unique signature for each image facilitates the identification of an individual by matching the signature to a database set of known of signatures (Yang et al., 2017). We previously reported the ability to identify pottery shards via a similar method of signature matching. Known pottery shards identified by archaeologists as representative samples from each time period and region can be analyzed, with vibrational signatures stored as a pottery vibrational signature library. Any new pottery shard can be compared to 


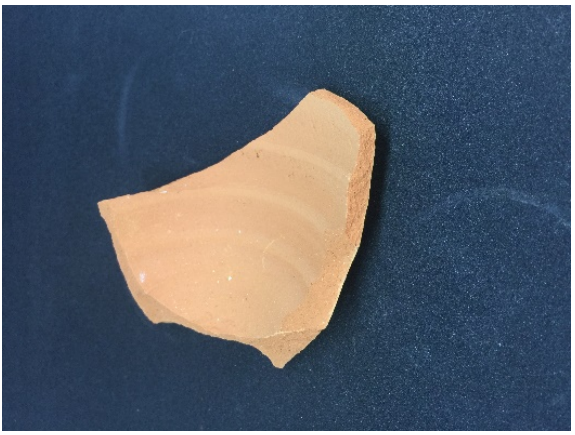

(a)

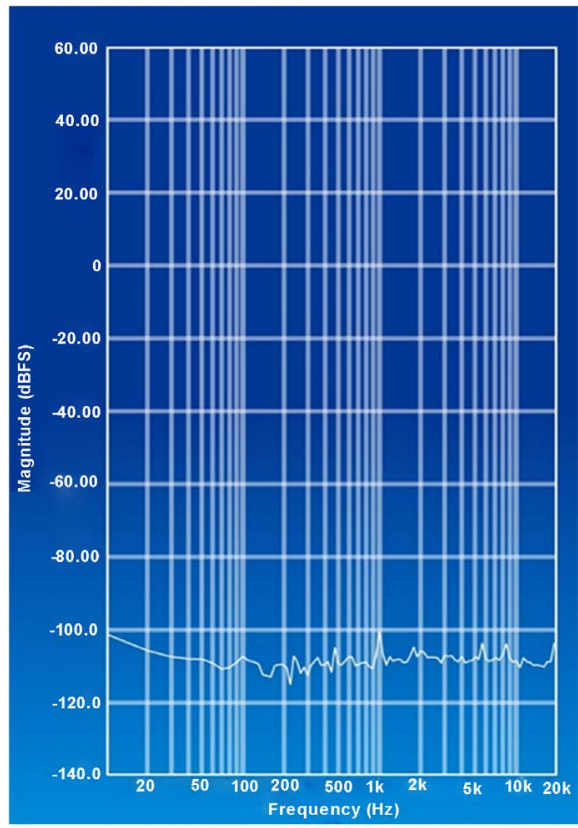

(b)

Figure 3. (a): A commercially available, common 20th century unglazed, lead-free pottery shard made in Mexico; (b) the corresponding vibrational profile, with spikes in the $9 \mathrm{~K}$, $6 \mathrm{~K}$, and $1 \mathrm{~K} \mathrm{~Hz}$ frequencies, and low frequency vibration in the below $10 \mathrm{~Hz}$ range.
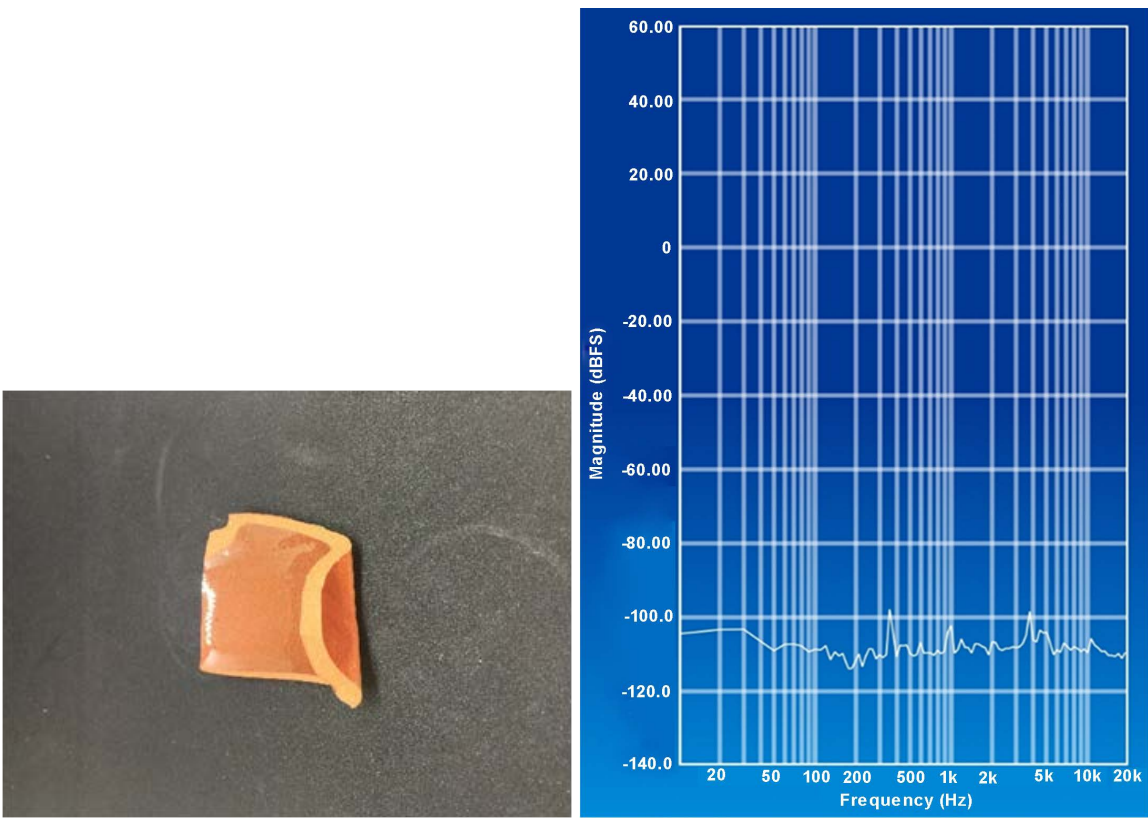

Figure 4. (a). A 20th century glazed pottery shard from Mexico; (b) the corresponding vibrational signature, with spikes in the $4 \mathrm{~K}, 1 \mathrm{~K}$, and $300 \mathrm{~Hz}$ range, and low frequency vibration, with a peak at the $30 \mathrm{~Hz}$ range.

the signature library to identity the new shard. The vibrational signatures will be compared by time analysis, amplitude analysis, dampening analysis, and frequency analysis in order to find the best match (LDS, 2003).

In this article we detail the experimental set-up and procedures necessary for 
such signature extraction. The system is designed to be portable so that it can be easily setup in a field lab for on-site, real-time analysis. The paper also shows the vibrational profiles from 20th century commercially available pottery shards, and such vibrational profiles can serve as calibration markers for researchers setting up a new system. This particular brand of pottery is common and commercially available. Once a new researcher has assembled the suggested setup for capturing vibrational signatures, one should start by recording the signature from the above standard pottery shard to ensure one can capture the standard wave form displayed in the article. We were able to observe unique vibrational patterns on two pottery shards manufactured in Mexico with similar soil composition, with the main difference being the firing technique and glazing finish. The fact that their differences can be reflected in their distinctive wave patterns is useful, and confirms the pathway of differentiating pottery from other areas.

In the current setup, all of the necessary equipment is commercially available, relatively affordable, and easy to assemble. It is the hope that, with this article, other archaeologists can benefit from the system and utilize the vibrational method of pottery shard identification.

Different energy sources can be used, and this paper details just three of the current options. Researchers are recommended to explore the vibrational patterns from other energy sources, such as microwaves, X-rays, or infrared waves. It is expected that each type of pottery will register a unique signature with each energy source. All of these vibrational waves can be compiled into a shared library to allow for collaboration and research cooperation.

\section{References}

Chen, B. (2017). Pottery Shard Analysis Using Matching Vibration Signatures. https://patents.google.com/patent/US20170307570A1/en

Eddine, A. K. J., Lenti, L., \& Semblat, J.-F. (2017). Vibrations in Soil: A spectral Prediction Method. Procedia Engineering, 199, 2675-2680. https://doi.org/10.1016/j.proeng.2017.09.546

Gazetas, G. (1982). Vibrational Characteristics of Soil Deposits with Variable Wave Velocity. International Journal for Numerical and Analytical Methods in Geomechanics, 6, 1-20. https://doi.org/10.1002/nag.1610060103

Historical Archaeology at the Florida Museum of Natural History (2018). Introduction to Ceramic Identification. http://www.floridamuseum.ufl.edu/

Hunt, A. (2017). The Oxford Handbook of Archaeological Ceramic Analysis. Oxford: Oxford University Press. https://doi.org/10.1093/oxfordhb/9780199681532.001.0001

Jacobsen, L. (1930). Motion of a Soil Subjected to a Simple Harmonic Ground Vibration. Bulletin of the Seismological Society of America, 20, 160-195.

Kitovas, V., Stelmokaitis, G., \& Doroševas, V. (2016). Investigation of Vibrations Influence on Clay Soil Parameters. Journal of Sustainable Architecture and Civil Engineering, 4, 60-67.

LDS Dactron Application Note (2003). Basics of Structural Vibrational Testing and Analysis.

https://www.calpoly.edu/ cbirdson/Publications/AN011\%20Basics\%20of\%20Structura 
1\%20Testing\%20\%20Analysis.pdf

MIT News (2014). Extracting Audio from Visual Information Algorithm Recovers Speech from the Vibrations of a Potato-Chip Bag Filmed through Soundproof Glass. http://news.mit.edu/2014/algorithm-recovers-speech-from-vibrations-0804

Parikh, S., Giyne, K. et al. (2014). Soil Chemical Insights Provided through Vibrational Spectroscopy. Advances in Agronomy, 126, 85-91.

https://doi.org/10.1016/B978-0-12-800132-5.00001-8

Renfrew, C., \& Bahn, P. (1991). Archaeology: Theories, Methods, and Practice. London: Thames \& Hudson.

Robertson, A. H. J., Shand, C., \& Perez-Fernandez, E. (2018). The Application of Fourier Transform Infrared, near Infrared and X-Ray Fluorescence Spectroscopy to Soil Analysis.

https://www.spectroscopyeurope.com/article/application-fourier-transform-infrared-n ear-infrared-and-x-ray-fluorescence-spectroscopy

Yang, J., Luo, L. et al. (2017). Nuclear Norm Based Matrix Regression with Applications to Face Recognition with Occlusion and Illumination Changes. IEEE Transactions on Pattern Analysis and Machine Intelligence, 39, 156-171.

https://doi.org/10.1109/TPAMI.2016.2535218 